\title{
Mejora en los calendarios de cultivo para agricultura de secano en ceja de selva
}

\section{Crop scheduling improvements for rainfed agriculture in the high jungle of Peru}

\author{
Enrique Meseth ; Jason C. S. Yu \\ National Sun Yat-Sen University, Department of Marine Environment and Engineering, Lien-Hai Road 70, 80424 \\ Kaohsiung, Taiwan.
}

Recibido 01 julio 2014. Aceptado 03 diciembre 2014.

\begin{abstract}
Resumen
Este trabajo tuvo como objetivo mejorar el manejo del agua para la agricultura en Vilcabamba y similares regiones en la ceja de selva, mediante eficientes calendarios de cultivo que permitan satisfacer las necesidades de agua utilizando agricultura de secano, a fin de maximizar el rendimiento de los cultivos. Para ello se llevó a cabo dos estudios de campo durante la temporada seca en septiembre de 2012 y la temporada de lluvias en febrero de 2013 para medir el caudal de ríos y canales; asimismo se analizaron 19 muestras del suelo, presentando texturas franco y franco arenoso. Se utilizó datos del clima, cultivos y suelo para calcular las necesidades de agua en los cultivos y las necesidades de riego con el programa Cropwat, indicando que la evapotranspiración de cultivos es baja debido a la humedad y temperatura fría. Los cultivos no requieren riego durante la temporada de lluvias, de diciembre a abril, sin embargo durante la temporada seca existen necesidades de riego, con una capacidad de flujo máximo de 1,72 $1 \mathrm{~s}^{-1}$ en mayo. La capacidad de flujo puede ser satisfecha por riachuelos y manantiales con caudales de 2 a $61 \mathrm{~s}^{-1}$ en la misma temporada. Los resultados de la investigación indican que es posible practicar la agricultura de secano, regando la tierra antes de la siembra para que el rendimiento de los cultivos no sea afectado, caso contrario algunas cosechas como verduras y papas se reducirían en un $4,7 \%$ y $1,4 \%$ respectivamente. Para minimizar estos efectos, se sugiere sembrar ambos cultivos un mes más tarde de lo acostumbrado, adaptando su periodo de crecimiento a la temporada de lluvias.
\end{abstract}

Palabras clave: calendarios de cultivo, necesidades de agua en los cultivos, agricultura de secano, manejo del agua, ceja de selva.

\section{Abstract}

This work was aimed to improve the water management for agriculture by applying efficient crop schedules in Vilcabamba and similar areas of the high jungle, which can satisfy most of the water requirements with rainfed agriculture to maximize the crops yield. For this purpose, two field practices were carried out during the dry (September 2012) and wet season (February 2013) to measure rivers and canals flows with the velocity/area method; 19 soil samples were collected on-site and analyzed, presenting prevalent sandy loam and loam textures. Cropwat program was used to estimate crop water requirements and scheme irrigation requirements, resulting in a maximum flow capacity of $1.72 \mathrm{l} \mathrm{s}^{-1}$ in May, during the dry season. The flow capacity can be satisfied, since small ditches convey approximately 2 to $6 \mathrm{l} \mathrm{s}^{-1}$ on the same season. The research findings indicate that rainfed farming can be practised, yet an initial pre-irrigation needs to be applied, for crops should not be water stressed. However, if soil is not pre-irrigated the production can be affected, with vegetables and potato crop yields being reduced by $4.7 \%$ and $1.4 \%$ respectively. To minimize these effects, both crops are suggested to be sowed one month later, adapting their growth period to the rainy season.
\end{abstract}

Keywords: crop scheduling, crop water requirements, rainfed irrigation, water management, high jungle agriculture.

\section{Introducción}

Vilcabamba enfrenta desafíos en la actualidad, puesto que el sector agrícola ha

\footnotetext{
* Autor para correspondencia

E-mail: enriquem3@ hotmail.com (E. Meseth).
}

sido descuidado por algunos gobiernos locales en la última década y como consecuencia, ha disminuido drásticamente 
su productividad y competitividad respecto a las regiones vecinas, quedando pocos agricultores locales que cultivan pequeñas parcelas con áreas que oscilan entre $0,25 \mathrm{y}$ 1 ha por agricultor. Asimismo, se ha realizado muy poca investigación de la agricultura en esta zona, de la cual cabe mencionar las tesis de Checya (2011) y Guzman (2008) de la Universidad San Antonio de Abad del Cusco. El valle de Vilcabamba se encuentra aislado en la ceja de selva y no se cuenta con transporte directo desde la ciudad del Cusco, incluso el acceso es interrumpido cuando ocurren lluvias extremas, ya que los huaycos dañan las carreteras al borde de las montañas del valle, siendo peligroso el tránsito.

Así, el presente estudio tiene como objetivo determinar si la gestión del agua en Vilcabamba es adecuada y sugerir mejoras en los calendarios de cultivo para incrementar su productividad.

\section{Materiales y métodos}

\subsection{Ubicación y prácticas de riego}

La región de Vilcabamba $\left(13^{\circ} 04^{\prime}-13^{\circ} 08^{\prime} \mathrm{S}\right.$, $\left.72^{\circ} 57^{\prime}-72^{\circ} 55^{\prime} \mathrm{O}\right)$ se encuentra en el flanco oriental de los Andes al sur de Perú, con una altitud promedio de $2900 \mathrm{msnm}$ inicia en los valles andinos a más de $4000 \mathrm{msnm}$ en la alta montaña, disminuyendo gradualmente hacia la ceja de selva en el norte a $1500 \mathrm{msnm}$ (Figura 1). El clima es semifrío, lluvioso y con invierno seco. La temporada seca en Vilcabamba es de mayo a noviembre, con escasas lluvias, las noches frías y días cálidos, mientras que la temporada de lluvias es húmeda, con precipitaciones desde diciembre hasta abril, y las noches son frías y lluviosas.

El paisaje está formado por colinas moderadas y escarpadas, con pendientes de $15 \%$ a más de $50 \%$, a lo largo de los valles aluviales del río Vilcabamba que fluye hacia el norte. En la Figura 2 se aprecia la topografía escarpada de esta región, obtenida de mapas del IGN (2010) y procesados en ESRICArcGis ${ }^{\mathrm{TM}}$ versión 10.

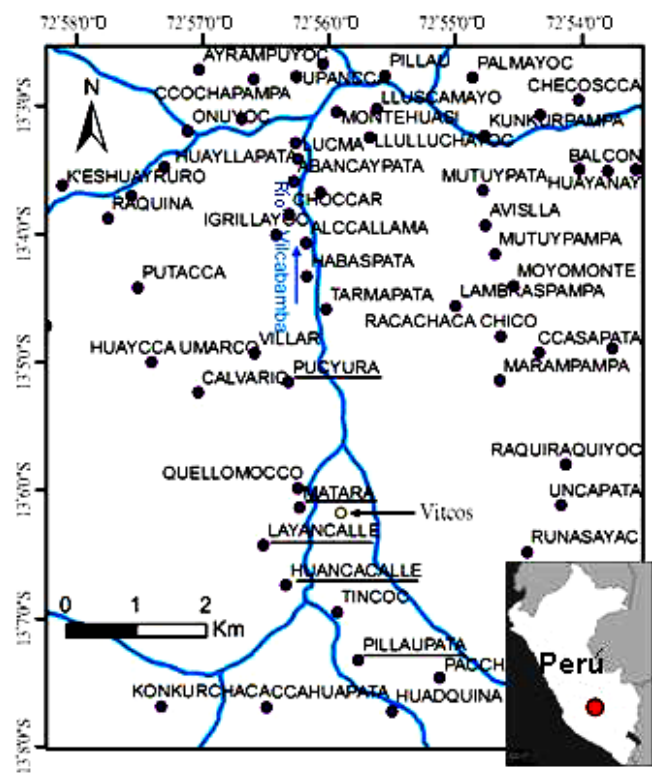

Figura 1. Valle de Vilcabamba con las principales localidades subrayadas, incluyendo el sitio arqueológico de Vitcos.

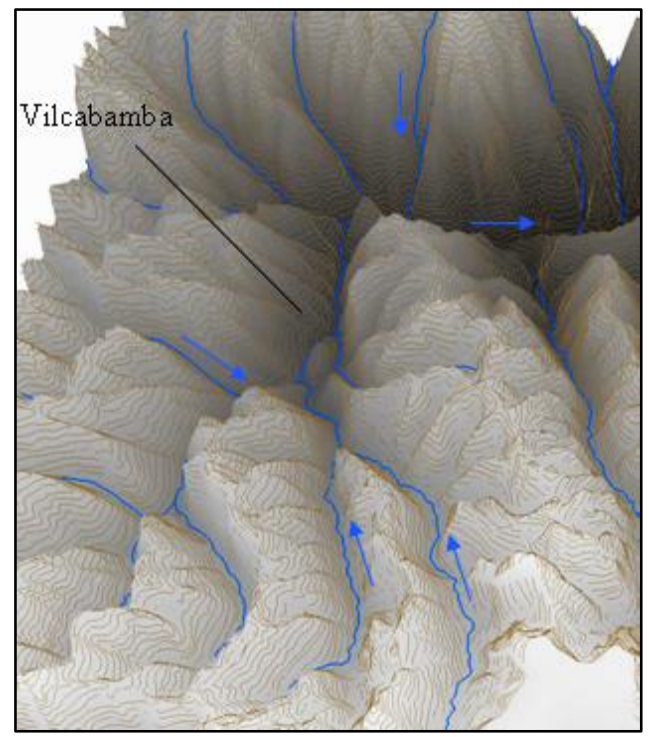

Figura 2. Topografía de Vilcabamba

Los riachuelos fluyen desde las cumbres de las montañas hasta los ríos en el fondo del valle, mientras que la mayor parte de la agricultura se practica a lo largo del valle y a una mayor altura que los ríos (Figura 3), ya que existen pocas tierras cultivables en los bordes de estos ríos y por tanto su agua casi no es utilizada. Por consiguiente, la mayoría de los agricultores practican la agricultura de secano, y pocos agricultores 
lo combinan con el riego por surcos. B. Cobos (comunicación personal, 15 de febrero, 2013) opina que este método no es muy eficiente ya que el agua se utiliza en los lugares de más altura en la época seca, reduciendo el flujo a medida que los riachuelos descienden la montaña. El riego por aspersión también se practica en pequeña escala y aún se utilizan técnicas rudimentarias como la labranza manual del suelo (Figura 4).

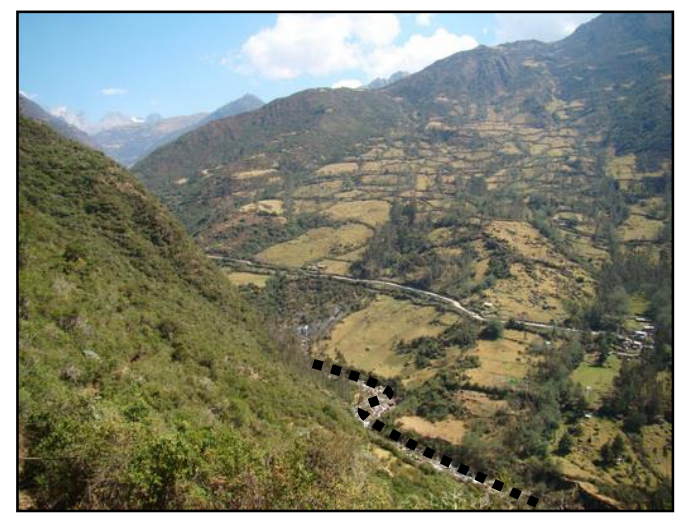

Figura 3. El valle de Vilcabamba, con el Río Cayco abajo (línea punteada).

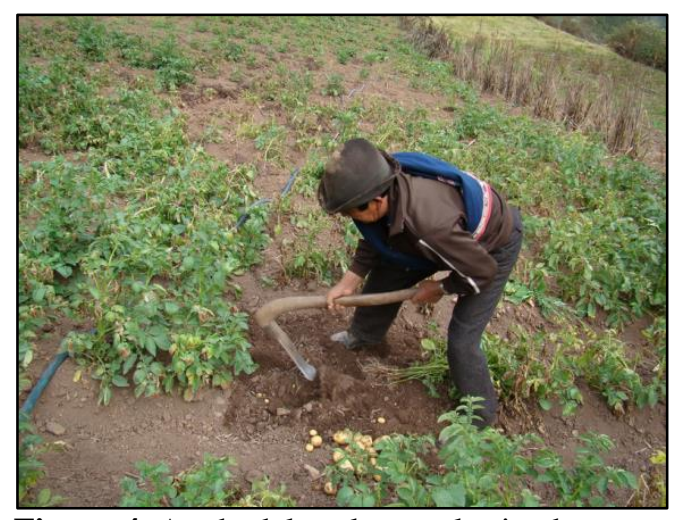

Figura 4. Arado del suelo para la siembra.

\subsection{Necesidades de agua en los cultivos}

Las necesidades de agua en los cultivos es la cantidad de agua necesaria para compensar la pérdida por evapotranspiración del campo cultivado. Mientras que las necesidades de riego representan la diferencia entre las necesidades de agua de los cultivos y la precipitación efectiva (Annandale et al., 2002). El programa Cropwat versión 8.0 se utilizó para estimar las necesidades de agua en los cultivos y el sistema de riego basado en el clima, el suelo y datos de los cultivos (Al-Najar, 2011). Se empleó el método de PenmanMontieth de FAO (El Nahry et al., 2010; Kuo et al., 2005) para calcular la evapotranspiración de referencia y la evapotranspiración del cultivo con las respectivas fórmulas:

$$
\begin{gathered}
\text { ETo }=\frac{0,408 \Delta(R n-G)+\gamma \frac{900}{T+273} u_{2}\left(e_{s}-e_{a}\right)}{\Delta+\gamma\left(1+0,34 u_{2}\right)} \\
\text { ETc }=\text { ETo } x \text { Kc }
\end{gathered}
$$

Donde ETo: evapotranspiración de referencia [mm $\mathrm{d}^{-1}$ ], $R n$ : radiación neta en la superficie de referencia [MJ $\mathrm{m}^{-2} \mathrm{~d}^{-1}$ ], $G$ : densidad del flujo del calor del suelo [MJ m-2 $\mathrm{d}^{-1}$ ], $T$ : temperatura del aire todos los días a $2 \mathrm{~m}$ de altura $\left[{ }^{\circ} \mathrm{C}\right], u_{2}$ : velocidad del viento a 2 $\mathrm{m}$ de altura $\left[\mathrm{m} \mathrm{s}^{-1}\right], e_{s}$ : presión de saturación de vapor $[\mathrm{kPa}], e_{a}$ : presión real de vapor $[\mathrm{kPa}], e_{s}-e_{a}$ : déficit de presión de saturación de vapor $[\mathrm{kPa}], \Delta$ : pendiente de la curva de presión de saturación de vapor $\left[\mathrm{kPa}{ }^{\circ} \mathrm{C}^{-1}\right], \gamma$ : constante psicrométrica $[\mathrm{kPa}$ $\left.{ }^{\circ} \mathrm{C}^{-1}\right]$, ETc: evapotranspiración del cultivo $\left[\mathrm{mm} \mathrm{d}^{-1}\right]$ y $K c$ : coeficiente del cultivo [adimensional].

\subsection{Datos climáticos}

El valle de Vilcabamba carece de estaciones meteorológicas, por tanto se han utilizado los registros climáticos de las estaciones meteorológicas más cercanas, proporcionados por el Servicio Nacional de Meteorología e Hidrología - Sehamhi: Vilcabamba (4000 msnm) y Machu Picchu (2459 msnm). Estas estaciones se encuentran a $7 \mathrm{~km}$ al oeste y a $40 \mathrm{~km}$ al este del valle, respectivamente. Los tres sitios se ubican en la misma latitud de $13^{\circ} \mathrm{S}$ y el valle se encuentra dentro del rango de altitud de las estaciones.

La Estación de Vilcabamba cuenta con datos históricos diarios en los años 19641970, mientras que la estación de Machu Picchu cubre un periodo más amplio: 1964-1977 y 1998-2009. Una comparación directa entre ambas estaciones no sería adecuada para calcular el clima del valle de Vilcabamba, por lo tanto el procedimiento se dividió en dos etapas.

En la primera etapa, el clima promedio mensual correspondiente a las décadas de los 60 y 70 de las estaciones meteoroló- 
gicas fue relacionado con la altitud de los tres sitios, para la estimación del clima del valle de Vilcabamba en ese periodo. Esta comparación se debe al gradiente adiabático, donde una variable atmosférica (por ejemplo, temperatura) disminuye con la altitud (Glickman, 2000; Gardner et al., 2009). Entonces se aplicó la fórmula de interpolación lineal para la Tabla 1:

$$
x_{2}=\left[\left(e_{2}-e_{1}\right) *\left(x_{3}-x_{1}\right)\right] /\left(e_{3}-e_{1}\right)+x_{1}
$$

\section{Tabla 1}

Determinación de la temperatura del Valle de Vilcabamba mediante la interpolación lineal de las estaciones meteorológicas con la altitud (décadas de los 60 y 70)

\begin{tabular}{lcc}
\hline Sitio & \multicolumn{1}{c}{ Altitud } & Temperatura \\
\hline Estación Machu Picchu & $e_{1}=2459 \mathrm{~m}$ & $x_{1}$ \\
Valle de Vilcabamba & $e_{2}=2900 \mathrm{~m}$ & $x_{2}$ \\
Estación Vilcabamba & $e_{3}=4000 \mathrm{~m}$ & $x_{3}$ \\
\hline
\end{tabular}

En la segunda etapa se utilizó una regla de tres simple para relacionar los resultados del valle de Vilcabamba con el mismo periodo climático de la Estación Machu Picchu (1964-1977), versus los registros totales de 26 años de la misma estación (1964-1977 y 1998-2009). Con este procedimiento se estimó el clima moderno en el valle de Vilcabamba $\left(t_{2}\right)$ con la fórmula detallada a continuación y la Tabla 2.

$$
t_{2}=\left(x_{2} * t_{1}\right) / x_{1}
$$

El procedimiento descrito fue aplicado para calcular la temperatura, la humedad y la velocidad del viento. Las horas de sol están relacionadas con la radiación solar, que también juega un papel importante en la evapotranspiración del cultivo. Los registros de horas de sol se obtuvieron de las estaciones meteorológicas vecinas de la base de datos climáticos Climwat versión 2.0 de la Organización de las Naciones Unidas para la Alimentación y la Agricultura - FAO: Ayacucho y Abancay, a unos $140 \mathrm{~km}$ al oeste y $60 \mathrm{~km}$ al sur del área de estudio, respectivamente, las cuales se promediaron para estimar la duración diaria del sol para cada mes.

\section{Tabla 2}

Determinación de la temperatura moderna del Valle de Vilcabamba mediante regla de tres simple, relacionada con la Estación de Machu Picchu (26 años de datos climáticos)

\begin{tabular}{lcc}
\hline \multirow{2}{*}{ Sitio } & \multicolumn{2}{c}{ Registros climáticos (años) } \\
\cline { 2 - 3 } & 1964-1977 & 1964-1977, \\
& & $1998-2009$ \\
\hline Estación Machu Picchu & $x_{1}$ & $t_{1}$ \\
Valle de Vilcabamba & $x_{2}$ & $t_{2}$ \\
\hline
\end{tabular}

La precipitación es el principal contribuyente para satisfacer las necesidades de agua en los cultivos en el área de estudio. Debido a la falta de registros de precipitaciones en esta área, se utilizaron los datos históricos de las estaciones de Senamhi y el procedimiento de dos etapas descrito anteriormente, obteniéndose la precipitación total mensual en el valle de Vilcabamba. Luego se estimó que los cultivos utilizan el $75 \%$ de la precipitación total, llamada precipitación efectiva (Instituto de Manejo del Agua y Medio Ambiente - IMA, 2010), esto se debe a pérdidas de lluvia por escorrentía superficial o infiltración por debajo de la zona de las raíces (Vallet et al., 2013).

\subsection{Propiedades del suelo}

El IMA (2005) describe la taxonomía de suelos de Vilcabamba como orthents de la clasificación del Departamento de Agricultura de EE.UU. - USDA, equivalente al tipo regosol de FAO. Checya (2011) analizó dos muestras en un área vecina obteniendo los siguientes resultados: un $\mathrm{pH}$ neutro que va desde 6,8 hasta 7,2 , bajo en nitrógeno con $0,26 \mathrm{ppm}$ en promedio, muy alto en fósforo en la primera muestra (102,5 ppm) y de concentración media en la segunda $(28,3$ ppm), muy alto en potasio con 812,5 ppm en promedio, un rango medio de materia orgánica $(5,22 \%)$ en promedio, conductividad eléctrica muy baja (CE) de $0,67 \mathrm{mS}$ $\mathrm{m}^{1}$ en promedio, y texturas de suelo franco y franco arcillo arenoso. El autor principal analizó 19 muestras del suelo de Vitcos en el valle de Vilcabamba, con texturas de suelo franco arcillo arenoso, franco limoso 
y principalmente franco arenoso y franco, de colores marrón a marrón rojizo.

De este modo, el suelo de Vilcabamba se relaciona con la categoría de tierra franco rojiza (red loamy soil) del Cropwat, con una humedad disponible total de 180 $\mathrm{mm} / \mathrm{m}$ de profundidad y una velocidad máxima de infiltración de $40 \mathrm{~mm} /$ día. Con esta información se ha estimado las necesidades de riego.

\subsection{Patrón de cultivo y necesidades del sistema de riego}

G. Santa Cruz (comunicación personal, 17 de septiembre, 2012) brindó información relacionada a tipos de cultivos, rendimiento y calendarios de cultivo en el valle, estimándose un patrón de cultivo de las cosechas principales (Clarke et al., 2005; 2010). Poco se ha investigado de la aislada Vilcabamba, sin embargo algunos libros y tesis sobre esta región han sido consultados.

Las necesidades del sistema de riego se refieren al suministro de agua para todo el área de regadío. Esto se obtiene mediante fórmulas secuenciales en Cropwat a partir de las necesidades de riego (IWR: Irrigation water requirements en inglés) en [mm mes $\left.{ }^{-1}\right]$ para un cultivo determinado: $I W R=$ ETc - precipitación efectiva

Luego, las necesidades netas del sistema de riego son calculadas, multiplicando las necesidades de riego de cada cultivo por el porcentaje de la superficie sembrada:

Necesidades netas del sistema de riego $=I W R$

* Porcentaje de la superficie sembrada

A continuación, las necesidades netas del sistema de riego son multiplicadas por la eficiencia del sistema de riego, expresadas en $\left[\mathrm{mm} \mathrm{d}^{-1}\right]$ o $\left[1 \mathrm{~s}^{-1} \mathrm{ha}^{-1}\right]$, obteniendo las necesidades brutas del sistema de riego. Por lo tanto el total de agua requerida para el riego es:

Necesidades brutas del sistema de riego $=$ Necesidades netas del sistema de riego x 100/e

La eficiencia del sistema de riego (e) se calcula con la fórmula:

$$
e=\left(e_{c} \times e_{a}\right) / 100
$$

Donde $e_{c}$ es la eficiencia del transporte de agua (por ejemplo canales) y $e_{a}$ es la eficiencia de la aplicación del agua en el campo.

Por último, la capacidad de flujo $(Q)$, expresada en $\left[\begin{array}{ll}1 & \mathrm{~s}^{-1}\end{array}\right]$ o $\left[\begin{array}{ll}\mathrm{m}^{3} & \mathrm{~s}^{-1}\end{array}\right]$, es la cantidad de agua requerida por toda la superficie cultivada y es el resultado de las necesidades brutas del sistema de riego multiplicado por el total del área cultivada:

$Q\left(l s^{-1}\right)=$ Necesidades brutas del sistema de riego $\left(l s^{-1} h a^{-1}\right)$ x Área total cultivada (ha)

\subsection{Hidrología}

La Estación hidrométrica ' $\mathrm{km} 105$ ' mide el caudal del río Vilcanota, uno de los principales ríos al sur de los Andes del Perú. La estación se sitúa $35 \mathrm{~km}$ al este de Vilcabamba y $10 \mathrm{~km}$ al noroeste de Machu Picchu. Datos mensuales de flujo mínimo, promedio y máximo han sido registrados por la Empresa de Generación Eléctrica Machupicchu S.A. - EGEMSA (2008) desde 1958 hasta 2007, excluyendo los años 1962, 1963 y 2000 (47 años en total). Estos datos han sido relacionados con la precipitación mensual de la Estación meteorológica proporcionados por SENAMHI.

Asimismo, se realizaron dos estudios de campo en Vilcabamba en la temporada seca (septiembre de 2012) y en la temporada de lluvias (febrero de 2013), a fin de medir el caudal de los cuerpos de agua de la zona y determinar si las necesidades brutas del sistema de riego son satisfechas. Por lo tanto el flujo de descarga $(Q)$ de ríos, riachuelos, canales y manantiales fue estimado con el método de velocidad/área, mediante la medición de la velocidad media de flujo $(V)$ y el área de sección transversal $(A)$, utilizando la siguiente fórmula:

$$
Q\left(m^{3} s^{-1}\right)=A\left(m^{2}\right) x V\left(m s^{-1}\right)
$$

La velocidad se calculó midiendo el tiempo que tarda un objeto flotante en recorrer una determinada distancia corriente abajo. 


\section{Resultados y discusión}

\subsection{Temperatura}

La temperatura de la zona de estudio se estimó en dos etapas descritas en la subsección de datos climáticos de materiales y métodos. La temperatura del valle de Vilcabamba dentro del periodo 1964-1977 se ilustran en la Tabla 3. La Tabla 4 muestra el resultado final de las temperaturas mínimas y máximas mensuales $\left({ }^{\circ} \mathrm{C}\right)$ del valle de Vilcabamba, obtenido de los datos históricos totales (1964-1977, 19982009). Las temperaturas mínimas en el valle de Vilcabamba se producen en las noches, mientras que las temperaturas máximas son durante el día. La temperatura mínima promedio estimada es de $6,5^{\circ} \mathrm{C}$ en julio, llegando a una máxima promedio de $19,9^{\circ} \mathrm{C}$ en agosto (Tabla 4); mientras que la temperatura media mensual es casi constante durante todo el año, de $13^{\circ} \mathrm{C}$ a $14^{\circ} \mathrm{C}$.

\subsection{Humedad y velocidad del viento}

Los resultados mensuales de humedad oscilaron entre $85 \%$ a $93 \%$, con un promedio anual de $89 \%$. Mientras que la velocidad del viento mensual varió de 2,9 a $3,6 \mathrm{~m} / \mathrm{s}$, con un promedio anual de $3,2 \mathrm{~m} / \mathrm{s}$.

\subsection{Duración del sol}

La Tabla 5 muestra el promedio de horas diarias de sol al mes de las estaciones meteorológicas de Abancay y Huamanaga obteniendo resultados para el valle de Vilcabamba, con un promedio de 6,9 horas de sol al mes.

\subsection{Evapotranspiración de referencia}

La evapotranspiración de referencia (ETo) resulta en una mínima de $2,71 \mathrm{~mm} d^{-1}$ en mayo, una máxima de hasta $3,40 \mathrm{~mm}^{-1}$ en noviembre (Figura 5) y un promedio anual de 2,97 $\mathrm{mm} d^{-1}$. Estos valores corresponden a regiones húmedas tropicales, con climas frío-templados, presentando una buena correlación con el lugar de estudio. En la Tabla 6 se detalla el clima, la radiación y la evapotranspiración de referencia mensuales.

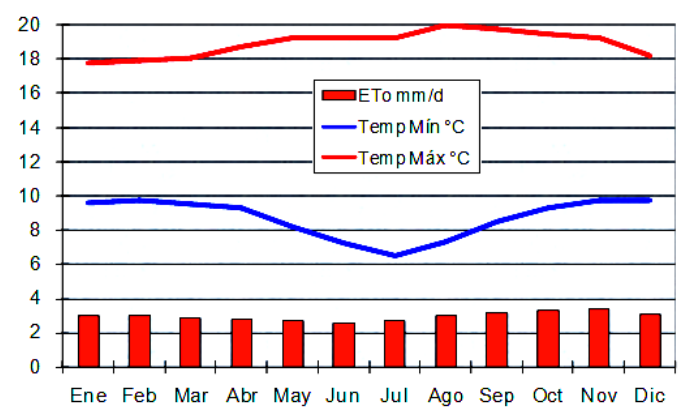

Figura 5. Temperatura mensual (máxima y mínima) y evapotranspiración de referencia (ETo).

\section{Tabla 6}

Clima y evapotranspiración de referencia (ETo) en el valle de Vilcabamba, Perú (Altitud: 2900 m; Latitud: $13,10{ }^{\circ} \mathrm{S}$; Longitud: $72,93{ }^{\circ} \mathrm{O}$ )

\begin{tabular}{lccccccc}
\hline Mes & $\begin{array}{c}\text { Temp } \\
\text { Mín } \\
\left({ }^{\circ} \mathrm{C}\right)\end{array}$ & $\begin{array}{c}\text { Temp } \\
\text { Máx } \\
\left({ }^{\circ} \mathrm{C}\right)\end{array}$ & $\begin{array}{c}\text { Hume- } \\
\text { dad })\end{array}$ & $\begin{array}{c}\text { Viento } \\
(\mathrm{m} / \mathrm{s})\end{array}$ & $\begin{array}{c}\text { Horas } \\
\text { de sol }\end{array}$ & $\begin{array}{c}\text { Rad } \\
\left(\mathrm{MJ} / \mathrm{m}^{2} / \mathrm{d}\right)\end{array}$ & $\begin{array}{c}\text { ETo } \\
(\mathrm{mm} / \mathrm{d})\end{array}$ \\
\hline Ene & 9,6 & 17,7 & 93 & 3,0 & 6,1 & 19,8 & 3,02 \\
Feb & 9,7 & 17,9 & 93 & 2,9 & 6,0 & 19,5 & 2,99 \\
Mar & 9,5 & 18,0 & 92 & 2,9 & 5,7 & 18,2 & 2,82 \\
Abr & 9,3 & 18,7 & 91 & 3,0 & 6,9 & 18,3 & 2,80 \\
May & 8,2 & 19,2 & 88 & 2,9 & 7,6 & 17,3 & 2,71 \\
Jun & 7,2 & 19,2 & 87 & 3,1 & 7,8 & 16,5 & 2,58 \\
Jul & 6,5 & 19,2 & 85 & 3,3 & 7,6 & 16,7 & 2,68 \\
Ago & 7,3 & 19,9 & 85 & 3,6 & 7,5 & 18,3 & 3,01 \\
Sep & 8,5 & 19,7 & 88 & 3,6 & 7,1 & 19,6 & 3,15 \\
Oct & 9,3 & 19,4 & 89 & 3,5 & 7,2 & 21,0 & 3,33 \\
Nov & 9,7 & 19,2 & 90 & 3,6 & 7,4 & 21,7 & 3,40 \\
Dic & 9,7 & 18,2 & 92 & 3,1 & 6,2 & 19,9 & 3,10 \\
\hline Prom & 8,7 & 18,9 & 89 & 3,2 & 6,9 & 18,9 & 2,97 \\
\hline
\end{tabular}

\subsection{Precipitación}

La precipitación en el valle de Vilcabamba resulta en $1820 \mathrm{~mm}$ al año, obteniendo una precipitación efectiva anual de $1365 \mathrm{~mm}$ que utilizan los cultivos. Ver detalles en la sub-sección de datos climáticos de materiales y métodos. La precipitación total y efectiva mensual se ilustra en la Figura 6.

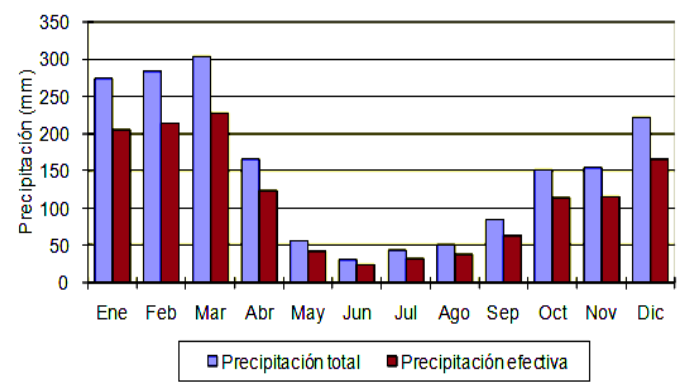

Figura 6. Precipitación total y precipitación efectiva $(\mathrm{mm})$ en el valle de Vilcabamba. 


\section{Tabla 3}

Temperaturas mínimas y máximas $\left({ }^{\circ} \mathrm{C}\right)$ promedias mensuales (años 1964-1977) para el valle de Vilcabamba (fila central), obtenidas de datos de las estaciones meteorológicas de Machu Picchu y Vilcabamba, interpolados por sus respectivas altitudes

\begin{tabular}{|c|c|c|c|c|c|c|c|c|c|c|c|c|c|c|c|c|c|c|c|c|c|c|c|c|c|c|c|c|}
\hline \multirow{2}{*}{ Sitio } & \multirow{2}{*}{$\begin{array}{l}\text { Altitud } \\
\text { (msnm) }\end{array}$} & \multirow[b]{2}{*}{ Años } & \multicolumn{2}{|c|}{ Ene } & \multicolumn{2}{|c|}{ Feb } & \multicolumn{2}{|c|}{ Mar } & \multicolumn{2}{|c|}{ Abr } & \multicolumn{2}{|c|}{ May } & \multicolumn{2}{|c|}{ Jun } & \multicolumn{2}{|c|}{ Jul } & \multicolumn{2}{|c|}{ Ago } & \multicolumn{2}{|c|}{ Sep } & \multicolumn{3}{|c|}{ Oct } & \multirow{2}{*}{$\begin{array}{l}\text { Nov } \\
\text { Máx }\end{array}$} & \multicolumn{2}{|c|}{ Dic } & \multicolumn{2}{|c|}{ Promedio } \\
\hline & & & Mín & Máx & Mín & Máx & Mín & Máx & Mín & Máx & Mín & Máx & Mín & Máx & Mín & Máx & Mín & Máx & Mín & Máx & Mín & Máx & Mín & & Mín & Máx & Mín & Máx \\
\hline Estación Machu Picchu & 2459 & 1964-1977 & 11,1 & 19,5 & 11,2 & 19,4 & 10,8 & 20,3 & 10,5 & 20,9 & 9,2 & 21,6 & 8,5 & 21,8 & 7,9 & 21,5 & 8,7 & 22,0 & 10,1 & 21,7 & 10,9 & 21,7 & 11,2 & 21,3 & 11,1 & 20,4 & 10,1 & 21,0 \\
\hline Valle de Vilcabamba & 2900 & Interpolación & 9,3 & 17,4 & 9,5 & 17,5 & 9,2 & 18,1 & $\mathbf{8 , 8}$ & 18,6 & $\mathbf{7 , 6}$ & 19,2 & 6,8 & 19,4 & 6,1 & 19,3 & 6,9 & 19,7 & 8,3 & 19,3 & 9,1 & 19,2 & 9,5 & 18,9 & $\mathbf{9 , 4}$ & 18,0 & $\mathbf{8 , 4}$ & 18,7 \\
\hline Estación Vilcabamba & 4000 & 1964-1970 & 5,0 & 12,1 & 5,2 & 12,8 & 5,3 & 12,5 & 4,7 & 12,6 & 3,7 & 13,2 & 2,4 & 13,4 & 1,8 & 13,6 & 2,5 & 14,0 & 3,9 & 13,1 & 4,6 & 13,1 & 5,2 & 12,9 & 5,1 & 12,1 & 4,1 & 12,9 \\
\hline
\end{tabular}

\section{Tabla 4}

Temperaturas mínimas y máximas mensuales $\left({ }^{\circ} \mathrm{C}\right)$ en el valle de Vilcabamba (resultados en última fila). Datos del periodo 1964-1977 de la Estación de Machu Picchu y el Valle de Vilcabamba, multiplicados con regla de tres por los datos históricos totales de Machu Picchu (1964-1977, 1998-2009)

\begin{tabular}{|c|c|c|c|c|c|c|c|c|c|c|c|c|c|c|c|c|c|c|c|c|c|c|c|c|c|c|c|}
\hline \multirow{2}{*}{ Sitio } & \multirow{2}{*}{ Años } & \multicolumn{2}{|c|}{ Ene } & \multicolumn{2}{|c|}{$\mathrm{Feb}$} & \multicolumn{2}{|c|}{ Mar } & \multicolumn{2}{|c|}{ Abr } & \multicolumn{2}{|c|}{ May } & \multicolumn{2}{|c|}{ Jun } & \multicolumn{2}{|c|}{ Jul } & \multicolumn{2}{|c|}{ Ago } & \multicolumn{2}{|c|}{ Sep } & \multicolumn{2}{|c|}{ Oct } & \multicolumn{2}{|c|}{ Nov } & \multicolumn{2}{|c|}{ Dic } & \multicolumn{2}{|c|}{ Promedio } \\
\hline & & Mín & Máx & Mín & Máx & Mín & Máx & Mín & Máx & Mín & Máx & Mín & Máx & Mín & Máx & Mín & Máx & Mín & Máx & Mín & Máx & Mín & Máx & Mín & Máx & Mín & Máx \\
\hline \multirow[b]{2}{*}{ Estación Machu Picchu } & 1964-1977 & 11,1 & 19,5 & 11,2 & 19,4 & 10,8 & 20,3 & 10,5 & 20,9 & 9,2 & 21,6 & 8,5 & 21,8 & 7,9 & 21,5 & 8,7 & 22,0 & 10,1 & 21,7 & 10,9 & 21,7 & 11,2 & 21,3 & 11,1 & 20,4 & 10,1 & 21,0 \\
\hline & $\begin{array}{l}\text { 1964-1977, } \\
1998-2009\end{array}$ & 11,3 & 19,8 & 11,4 & 19,8 & 11,1 & 20,2 & 11,0 & 21,1 & 9,9 & 21,6 & 9,0 & 21,7 & 8,3 & 21,5 & 9,1 & 22,2 & 10,4 & 22,2 & 11,1 & 21,9 & 11,4 & 21,7 & 11,4 & 20,5 & 10,5 & 21,2 \\
\hline \multirow{2}{*}{ Valle de Vilcabamba } & 1964-1977 & 9,3 & 17,4 & 9,5 & 17,5 & 9,2 & 18,1 & 8,8 & 18,6 & 7,6 & 19,2 & 6,8 & 19,4 & 6,1 & 19,3 & 6,9 & 19,7 & 8,3 & 19,3 & 9,1 & 19,2 & 9,5 & 18,9 & 9,4 & 18,0 & 8,4 & 18,7 \\
\hline & Regla de tres & 9,6 & 17,7 & 9,7 & 17,9 & 9,5 & 18,0 & 9,3 & 18,7 & 8,2 & 19,2 & 7,2 & 19,2 & 6,5 & 19,2 & 7,3 & 19,9 & 8,5 & 19,7 & 9,3 & 19,4 & 9,7 & 19,2 & 9,7 & 18,2 & 8,7 & 18,9 \\
\hline
\end{tabular}

\section{Tabla 5}

Horas diarias de sol al mes en el valle de Vilcabamba, basado en promedios de las estaciones de Abancay y Huamanga

\begin{tabular}{lccccccccccccc}
\hline Sitio & Ene & Feb & Mar & Abr & May & Jun & Jul & Ago & Sep & Oct & Nov & Dic & Promedio \\
\hline Estación Abancay & 5,7 & 5,6 & 5,7 & 6,9 & 7,6 & 7,7 & 7,8 & 8,0 & 6,9 & 7,2 & 7,0 & 5,7 & $\mathbf{6 , 8}$ \\
Estación Huamanga & 6,4 & 6,3 & 5,7 & 6,9 & 7,6 & 7,8 & 7,3 & 7,0 & 7,2 & 7,2 & 7,7 & 6,7 & \\
\hline Valle de Vilcabamba & $\mathbf{6 , 1}$ & $\mathbf{6 , 0}$ & $\mathbf{5 , 7}$ & $\mathbf{6 , 9}$ & $\mathbf{7 , 6}$ & $\mathbf{7 , 8}$ & $\mathbf{7 , 6}$ & $\mathbf{7 , 5}$ & $\mathbf{7 , 1}$ & $\mathbf{7 , 2}$ & $\mathbf{7 , 4}$ & $\mathbf{6 , 2}$ & \\
\hline
\end{tabular}




\subsection{Patrón de cultivo}

La papa y el maíz blanco son los principales cultivos en la zona de estudio, con una superficie cultivada de 15 ha cada uno, cubriendo el $82 \%$ de la superficie total, con un rendimiento de 8 y 3 t/ha respectivamente. Otros cultivos son las arvejas, ocupando el $8 \%$ de la superficie cultivada ( $3 \mathrm{ha}$ ) y un rendimiento de 1,3 $\mathrm{t} / \mathrm{ha}$; las habas y verduras ocupan 2 ha y $5 \%$ de la superficie cultivada cada uno, con un rendimiento de 1 y 4 t/ha respectivamente. Las verduras cultivadas son variadas, e incluyen la col, la lechuga, la zanahoria y el tomate, utilizados principalmente para consumo personal. La Figura 7 muestra el patrón de cultivo con calendarios de siembra y distribución de los cultivos.

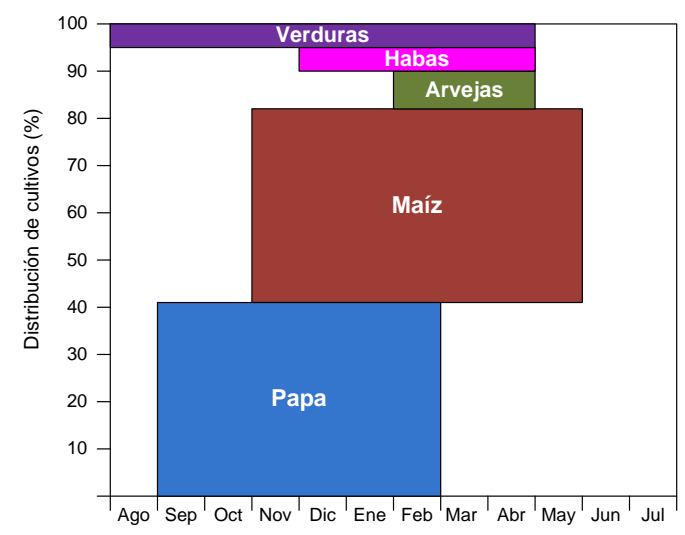

Figura 7. Patrón de cultivo.

\subsection{Necesidades de agua en los cultivos y necesidades de riego}

Los resultados del balance hídrico establecieron que las necesidades de agua de la papa son $38,5 \mathrm{~mm}$, con necesidades de riego de $2,3 \mathrm{~mm}$ en noviembre (etapa media de crecimiento); las necesidades de agua del maíz son $56,1 \mathrm{~mm}$, con necesidades de riego de $14,1 \mathrm{~mm}$ en mayo (etapa final de crecimiento); las necesidades de agua de las arvejas son 3 $\mathrm{mm}$, con necesidades de riego de $3 \mathrm{~mm}$ en mayo (etapa final de crecimiento); las habas no necesitan riego en todo su ciclo de crecimiento; las necesidades de agua de las verduras son $64,9 \mathrm{~mm}$ en agosto y 43,7 $\mathrm{mm}$ en septiembre, con necesidades de riego de $26,4 \mathrm{~mm}$ y $6,5 \mathrm{~mm}$ en los meses respectivos (etapa inicial de crecimiento). Durante la temporada de lluvias intensas (diciembre a abril) las necesidades de agua de los cultivos son satisfechas por las precipitaciones y no hay necesidad de riego.

\subsection{Necesidades del sistema de riego}

El sistema de riego presenta déficit de agua en mayo, agosto, septiembre y noviembre, requiriendo una capacidad de flujo de 1,72 , $0,38,0,09$ y $0,271 \mathrm{~s}^{-1}$ respectivamente, detallado en la Tabla 7. Por ejemplo en mayo, las necesidades de riego para el maíz y las arvejas son $14,1 \mathrm{~mm}^{-\mathrm{mes}^{-1}}$ y 3 $\mathrm{mm}$ mes $^{-1}$ respectivamente. Las necesidades de riego de cada cultivo multiplicado por la superficie sembrada resulta en necesidades netas de riego de $0,2 \mathrm{~mm} /$ día para ese mes:

Necesidades netas de riego $=14,1 \times 41 \%+3 \times 8 \%$ $=6,02 \mathrm{~mm} \mathrm{mes}^{-1}=0,2 \mathrm{~mm} \mathrm{dí}^{-1}$

La eficiencia del sistema de riego $(e)$ se estimó en 50\%, considerando una eficiencia de conducción $\left(e_{c}\right)$ de $75 \%$ para canales de suelo franco de distancia media (200$2000 \mathrm{~m}$ ), y una eficiencia de aplicación en el campo $\left(e_{a}\right)$ de $67,5 \%$ considerando surcos y aspersores con eficiencias de 60\% y $75 \%$ respectivamente. Por tanto las necesidades brutas del sistema de riego son:

Necesidades brutas del sistema de riego $=$ $2 \mathrm{~mm} \mathrm{~d}^{-1} \times 100 / 50=0,4 \mathrm{~mm} \mathrm{~d}^{-1}=0,046 \mathrm{l}$ $s^{-1} h a^{-1}$

Finalmente, las necesidades brutas del sistema de riego en un área cultivada de 37 ha resultan en una capacidad de flujo (Q) de $1,72 \mathrm{ls}^{-1}$ en mayo, como se muestra a continuación. Una metodología similar fue utilizada para los otros meses con déficit de agua.

$$
Q=0,046 l s^{-1} h^{-1} \times 37 h a=1,72 l s^{-1}
$$


Tabla 7

Necesidades del sistema de riego en Vilcabamba

\begin{tabular}{|c|c|c|c|c|c|c|c|c|c|c|c|c|c|c|}
\hline Mes & Ene & Feb & Mar & Abr & May & Jun & Jul & Ago & Sep & Oct & Nov & Dic & & \\
\hline \multicolumn{15}{|l|}{ Déficit de precipitación } \\
\hline 1. Рapa & 0 & 0 & 0 & 0 & 0 & 0 & 0 & 0 & 0 & 0 & 2,3 & 0 & $41 \%$ & $\geq$ \\
\hline 2. Maíz & 0 & 0 & 0 & 0 & 14,1 & 0 & 0 & 0 & 0 & 0 & 0 & 0 & $41 \%$ & \\
\hline 3. Arvejas & 0 & 0 & 0 & 0 & 3 & 0 & 0 & 0 & 0 & 0 & 0 & 0 & $8 \%$ & 8 \\
\hline 4. Habas & 0 & 0 & 0 & 0 & 0 & 0 & 0 & 0 & 0 & 0 & 0 & 0 & $5 \%$ & 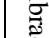 \\
\hline 5. Verduras & 0 & 0 & 0 & 0 & 0 & 0 & 0 & 26,4 & 6,5 & 0 & 0 & 0 & $5 \%$ & 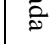 \\
\hline \multicolumn{15}{|l|}{ Área irrigada } \\
\hline (\% del área total) & 0 & 0 & 0 & 0 & 49 & 0 & 0 & 5 & 5 & 0 & 41 & 0 & & \\
\hline \multicolumn{15}{|c|}{ Necesidades netas del sistema de riego } \\
\hline en $\mathrm{mm} / \mathrm{mes}$ & 0 & 0 & 0 & 0 & 6,02 & 0 & 0 & 1,320 & 0,33 & 0 & 0,94 & 0 & & \\
\hline en $\mathrm{mm} / \mathrm{d}$ & 0 & 0 & 0 & 0 & 0,2 & 0 & 0 & 0,044 & 0,01 & 0 & 0,03 & 0 & $\mathrm{x} 2$ & (a) \\
\hline \multicolumn{15}{|c|}{ Necesidades brutas del sistema de riego } \\
\hline en $\mathrm{mm} / \mathrm{d}$ & 0 & 0 & 0 & 0 & 0,4 & 0 & 0 & 0,088 & 0,02 & 0 & 0,06 & 0 & x 0,116 & (b) \\
\hline en $1 /$ s/ha & 0 & 0 & 0 & 0 & 0,05 & 0 & 0 & 0,010 & 0 & 0 & 0,01 & 0 & x 37 & (c) \\
\hline Capacidad de flujo Q (1/s) & 0 & 0 & 0 & 0 & 1,72 & 0 & 0 & 0,38 & 0,09 & 0 & 0,27 & 0 & & \\
\hline
\end{tabular}

(a) Eficiencia de riego $e=100 / 50$

(b) Conversión de unidades

(c) Área total (ha).

\subsection{Hidrología}

Las precipitaciones son relacionadas con el caudal del río Vilcanota (subcapítulo 2.6 de materiales y métodos). Resultados en la Figura 8 muestran que la lluvia aumenta intensamente a partir de agosto $y$ septiembre, sin embargo el flujo del Río Vilcanota no se ve afectado en esos meses, sugiriendo que el suelo seco absorbe la mayor parte del agua de lluvias. Las lluvias continúan en octubre y el caudal comienza a aumentar, siguiendo el mismo patrón de las precipitaciones, esto indica que los suelos se han saturado y la lluvia se escurre hacia el río.

La precipitación influye en el caudal del río durante toda la temporada de lluvias, desde noviembre hasta febrero-marzo, para luego disminuir abruptamente de abril a junio, existiendo una estrecha correlación entre ambos. El flujo promedio del río Vilcanota es de $47,15 \mathrm{~m}^{3} \mathrm{~s}^{-1}$ en la temporada seca (mayo-noviembre), incrementando hasta $225,16 \mathrm{~m}^{3} \mathrm{~s}^{-1}$ en la temporada de lluvias (diciembre-abril), resultando en una variación de $478 \%$, casi 5 veces mayor.

La Tabla 8 brinda información del flujo de los principales ríos y algunos riachuelos de Vilcabamba, donde el caudal puede disminuir a menos de 13 veces de una temporada a otra.

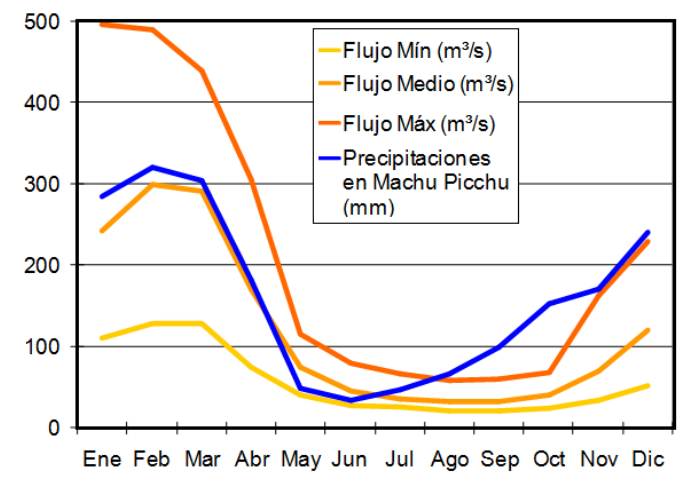

Figura 8. El flujo del río Vilcanota en relación a precipitaciones. Caudal promedio años 1958-2007 excluyendo 1962, 1963 y 2000 (EGEMSA, 2008); precipitaciones años 19641977 y 1998-2009 (SENAMHI, 2013).

Si se relacionan estos resultados con la capacidad de flujo del sistema, las necesidades de riego serían satisfechas, incluso durante la temporada seca, ya que en septiembre de 2012 las pequeñas acequias en los campos de cultivo transportaban 0,006 y $0,002 \quad \mathrm{~m}^{3} \quad \mathrm{~s}^{-1}$, cubriendo la máxima capacidad de flujo requerida $\left(0,0017 \mathrm{~m}^{3} \mathrm{~s}^{-1}\right)$. 
Tabla 8

Capacidad de flujo de ríos y canales en Vilcabamba, en temporada de lluvias y seca

\begin{tabular}{|c|c|c|c|c|c|c|c|c|}
\hline \multirow[b]{2}{*}{ Cuerpos de agua } & \multicolumn{4}{|c|}{ Febrero 2013 (temporada de lluvias) } & \multicolumn{4}{|c|}{ Septiembre 2012 (temporada seca) } \\
\hline & $\begin{array}{l}\text { Profun- } \\
\text { didad } \\
(\mathrm{m})\end{array}$ & $\begin{array}{l}\text { Ancho } \\
(\mathrm{m})\end{array}$ & $\begin{array}{l}\text { Velocidad } \\
(\mathrm{m} / \mathrm{s})\end{array}$ & $\begin{array}{l}\text { Flujo } \\
\left(\mathrm{m}^{3} / \mathrm{s}\right)\end{array}$ & $\begin{array}{c}\text { Profundi- } \\
\text { dad (m) }\end{array}$ & $\begin{array}{l}\text { Ancho } \\
(\mathrm{m})\end{array}$ & $\begin{array}{l}\text { Velocidad } \\
\text { (m/s) }\end{array}$ & $\begin{array}{l}\text { Flujo } \\
\left(\mathrm{m}^{3} / \mathrm{s}\right)\end{array}$ \\
\hline Río Cayara & 1,70 & 8,00 & 3,48 & 47,33 & 0,76 & 5,00 & 1,11 & 4,22 \\
\hline Río Cayco (sur de Vitcos) & 2,00 & 8,70 & 2,38 & 41,43 & & & & \\
\hline Río Cayco (oeste de Vitcos) - estimado & & & & 88,76 & & & & \\
\hline Río Pachaco (Andén) & 0,50 & 4,00 & 2,48 & 4,95 & 0,45 & 1,50 & 0,57 & 0,38 \\
\hline Río Habaspata (Pucyura) & 0,10 & 5,00 & 2,54 & 1,27 & & & & \\
\hline Canal Inca en Vitcos & 0,11 & 0,47 & 1,18 & 0,06 & & & & \\
\hline Acequia en Andén No.1 - Este de Vitcos & 0,15 & 0,80 & 1,27 & 0,15 & & & & \\
\hline Acequia en Anden No.3 - Este de Vitcos & 0,13 & 0,72 & 1,33 & 0,12 & 0,10 & 0,45 & 0,13 & 0,006 \\
\hline Manantial & & & & & 0,05 & 0,48 & 0,10 & 0,002 \\
\hline
\end{tabular}

Las simulaciones del presente estudio fueron realizadas en Cropwat, tomando como referencia estudios previos realizados por Clarke et al. (2005, 2010). Los resultados determinaron que es posible practicar agricultura de secano en Vilcabamba, sin embargo se recomienda aplicar un riego antes de la siembra, para que el rendimiento de los cultivos no sea afectado. Esto indica que los agricultores de estas regiones realizan una buena gestión en la programación de los calendarios de cultivo, beneficiándose de la temporada de lluvias. Sin embargo, existen necesidades brutas de riego del sistema en los meses de mayo, agosto, septiembre y noviembre, con capacidades de flujo de 1,72, 0,38, 0,09 y 0,27 $1 \mathrm{~s}^{-1}$ respectivamente. Mediciones de flujo in situ indican que la capacidad de flujo puede ser satisfecha por pequeñas acequias, incluso durante la temporada seca.

Por otro lado, si se practicase agricultura de secano sin riego previo a la siembra para humedecer la tierra, entonces el rendimiento de los cultivos se vería afectado. Como práctica general, la papa es sembrada a principios de septiembre en esta región -designando la fecha $1^{\circ}$ de septiembre para simulaciones en Cropwat, el agotamiento del suelo durante esta época del año sería de $65 \%$, con una humedad inicial disponible de $63 \mathrm{~mm} / \mathrm{m}$ de un total de $180 \mathrm{~mm} / \mathrm{m}$ para suelo franco, y por tanto el rendimiento de este cultivo se reduciría en un $1,4 \%$. Para verduras sembradas a inicios de agosto, el agotamiento del suelo sería del $80 \%$ debido a la temporada seca, con una humedad inicial disponible de $36 \mathrm{~mm} / \mathrm{m}$, y el rendimiento se reduciría en un $4,7 \%$. Por lo tanto se sugiere retrasar el calendario de cultivo de papas y verduras por un mes. Por ejemplo, si la papa se sembrase en octubre, su rendimiento no sería afectado, evitando una pérdida de producción de $1700 \mathrm{~kg}$; si las verduras se sembrasen en septiembre, la reducción del rendimiento sería sólo de $1,2 \%$, evitando una pérdida de $260 \mathrm{~kg}$. Considerar que los vegetales son cultivados en parcelas pequeñas para consumo personal, donde el agua es más fácil de administrar para el riego.

\section{Conclusiones}

Del presente estudio se concluye que el manejo del agua para la agricultura puede ser mejorado en Vilcabamba y en zonas de ceja de selva con altitudes similares, adaptando los calendarios de cultivo para obtener prácticas eficientes de agricultura de secano. La investigación determina que las necesidades de agua son bajas, favorecidas por el clima fresco y subhúmedo de Vilcabamba y debido a que la etapa media del crecimiento de los cultivos es programada durante la temporada de lluvias, para aprovechar las abundantes precipitaciones de diciembre a agosto, y conservar la humedad del suelo. Estas 
prácticas han sido transferidas empíricamente durante siglos en los Andes. Sin embargo, es necesario refinar los calendarios de cultivo cuando se aplica la agricultura de secano y sin riego antes de la siembra. En este aspecto, técnicas de simulación basadas en clima, suelo y datos de cultivos sugieren que los calendarios de algunos cultivos sean retrasados por un mes, a fin de maximizar el rendimiento y mitigar la escasez de agua para los cultivos. De ser viable, se recomienda el riego por aspersión, como una alternativa más eficiente a los canales. Este método de riego puede utilizarse para humedecer la tierra antes del arado y siembra en la temporada seca (Mayo a Agosto), para remediar el déficit de agua en los cultivos.

\section{Agradecimientos}

Se agradece el apoyo de las instituciones: National Sun Yat-Sen University, Department of Marine Environment and Engineering, Taiwán; Ingenieros para el Desarrollo - Engineers for Development INDEFOD, Perú. Asimismo, se agradece el valioso aporte de los especialistas: Ing. Zenón Huamán Gutiérrez, Director del Servicio Nacional de Meteorología e Hidrología - Senamhi Cusco, por proporcionar datos climáticos de la Estación de Machu Picchu; Sr. Grimaldo Santa Cruz Alvarez, Ex Teniente Alcalde y Regidor de la Municipalidad de Vilcabamba, por proporcionar información de cultivos de la zona de Vilcabamba; Sr. Benjamin Cobos Tejada, por brindar información acerca de los campos agrícolas en Vilcabamba; Dr. Derek Clarke, Profesor de University of Southampton (Inglaterra), por sus sugerencias acerca de las necesidades de riego; Luis Manuel Iriarte O'Brien, por el apoyo en estudios de campo en Vilcabamba; y Patricia Muñoz Tapia, por su apoyo logístico y trámites en la ciudad de Lima.

\section{Referencias bibliográficas}

Al-Najar, H. 2011. The integration of FAO-Cropwat model and GIS techniques for estimating irrigation water requirement and its application in the Gaza Strip. Natural Resources 2, 3: 146-154.

Annandale, J.; Jovanovic, N.; Benadé, N.; Allen, R. 2002. Software for missing data error analysis of PenmanMonteith reference evapotranspiration. Irrigation Science 21, 2: 57-67.
Checya, C. 2011. Diagnóstico agropecuario de la micro cuenca de Vilcabamba, Distrito de Vilcabamba, La Convención, Región Cusco. Tesis de bachillerato, Facultad de Agronomía y Zootecnia, Universidad Nacional de San Antonio de Abad del Cusco. Cusco, Perú.

Clarke, D.; Andrews, P.; Meseth, E.; Sala R.; Deom, J.M. 2010. Analysis of the hydraulics of the irrigation canals of Otar, Kazakhstan. Water Science \& Technology: Water Supply-WSTWS 10, 3: 453-461.

Clarke, D.; Sala R.; Deom, J.M.; Meseth, E. 2005. Reconstructing irrigation at Otrar Oasis, Kazakhstan AD 800-1700. Irrigation and Drainage 54: 375-388.

Empresa de Generación Eléctrica Machupicchu S.A. EGEMSA. 2008. Estudio de Impacto Ambiental de la Central Hidroeléctrica Santa Teresa. Capítulo III: Línea base ambiental. EGEMSA. Cusco, Perú.

El Nahry, A. H.; Ali, R. R.; El Baroudyc, A. A. 2010. An approach for precision farming under pivot irrigation system using remote sensing and GIS techniques. Agricultural Water Management 98: 517-551.

Gardner, A.S.; Sharp, M.J., Koerner, R.M.; Labine, C.; Boon, S.; Marshall, S.J.; Burgess, D.O.; Lewis, D. 2009. Near-surface temperature lapse rates over arctic glaciers and their implications for temperature downscaling. American Meteorological Society 22: 4281-4298.

Glickman, T. S. 2000. Glossary of Meteorology. American Meteorological Society 2: 855.

Guzman, D. 2008. Sondeo Rural Rápido en la Subcuenca de Vilcabamba del Distrito de Vilcabamba, La Convención, Cusco. Tesis de bachillerato, Facultad de Agronomía y Zootecnia, Universidad Nacional de San Antonio de Abad del Cusco. Cusco, Perú.

IGN - Instituto Geográfico Nacional. 2010. Mapas temáticos digitalizados del Distrito de Vilcabamba incluyendo topografía, hidrología, sitios arqueológicos y vegetación. Instituto Geográfico Nacional. Lima, Perú.

Instituto de Manejo del Agua y Medio Ambiente - IMA. 2010. Informe final de demanda hídrica actual y futura en la Región Cusco en el marco del programa de adaptación al cambio climático fase II y III - nivel regional. Proyecto Especial Instituto de Manejo del Agua y Medio Ambiente. IMA. Lima, Perú.

Kuo, S.F.; Ho, S.S.; Liu, C.W. 2005. Estimation irrigation water requirements with derived crop coefficients for upland and paddy crops in ChiaNan Irrigation Association, Taiwan. Agricultural Water Management 82: 433-451.

Servicio Nacional de Meteorología e Hidrología del Perú SENAMHI. 2013. Datos históricos meteorológicos e hídricos en Cusco. SENAMHI. Cusco, Perú.

Vallet, A.; Bertrand, C.; Mudry, J. 2013. Effective rainfall: a significant parameter to improve understanding of deep-seated rainfall triggering landslide - a simple computation temperature based method applied to Séchilienne unstable slope (French Alps). Hydrology and Earth System Sciences 10: 8945-8991. 